\title{
GATA-3 Stimulates Proliferation of Endometriotic Cells
}

\author{
Peng Chen ${ }^{1}$, Fang Ren ${ }^{1}$, Dan-Bo Wang ${ }^{1,2}$ \\ ${ }^{1}$ Department of Obstetrics and Gynecology, Shengjing Hospital of China Medical University, Shenyang, China \\ ${ }^{2}$ Department of Gynecology, Cancer Hospital of China Medical University, Shenyang, China
}

\begin{abstract}
Objectives: This study aimed to investigate whether there was a correlation between the expression of GATA binding protein 3 (GATA-3) and the proliferation of endometrium cells from women with endometriosis.

Material and methods: Endometrial tissue was collected from 20 women with endometriosis (experimental group) and 10 women without endometriosis (control group). The primary endometrial cells were then cultured and identified. Each group of cells were further divided into four groups. The control group was divided into control-no treatment (C-N), control-negative control (C-NC, transfected with a scrambled siRNA negative control), control-lentiviral transfection control (C-LC) and control-GATA-3 lentiviral transfection (C-G3); the experimental group was divided into experiment-no treatment (E-N), experiment-negative control (E-NC), experiment-lentiviral transfection control (E-LC) and experiment-siGATA-3 lentiviral transfection (E-siG3). RT-PCR and western blot were used respectively to detect the endometrium cells' GATA-3 mRNA and protein expression. Further, Cell Counting Kit-8 was used to measure the endometrium cells' proliferation.
\end{abstract}

Results: The relative expression of GATA-3 mRNA and protein in the E-N group was significantly higher than in the D-N group and E-siG3 group $(P<0.05)$. The relative cell proliferation rate in the $\mathrm{C}-\mathrm{G} 3$ group at $48 \mathrm{~h}, 72 \mathrm{~h}$ and $96 \mathrm{~h}$ was significantly higher than in the C-N, C-NC and C-LC groups $(P<0.05)$, while in the experimental group, the proliferation rate of the E-siG3 group was significantly lower than in the E-N, E-NC and E-LC groups $(P<0.05)$.

Conclusions: This study suggests the role of GATA-3 in promoting proliferation of endometrial cells; therefore, GATA-3 may play an essential role in the development of endometriosis.

Key words: endometriosis; GATA-3; endometriotic cell; proliferation

Ginekologia Polska 2019; 90, 6: 308-313

\section{INTRODUCTION}

Endometriosis, characterized by endometrial-like tissue and stroma outside the endometrial cavity, is known as a commonly encountered disease affecting $5-15 \%$ of women in their reproductive age [1]. Although $20-25 \%$ of women with endometriosis have no symptoms, patients may present with chronic pelvic pain, dysmenorrhea and infertility [2]. It has been nearly 90 years since this disease was initially described by Sampson [3], and several theories have been proposed to understand and explain the development of endometriosis [4]; however, the current understanding of the exact pathophysiology of endometriosis remains unclear.

Endometrial epithelial cells have been found by many recent studies working as a big source of cytokine production, which plays a very important role in the immune, menstrual and reproductive functions of endometrium $[5,6]$.
For instance, one study suggested that the imbalance of Thelper type 1 and 2 (Th1/Th2) cytokines are of great importance in the development of endometriosis [7]. Interestingly, in lymphocytes, GATA binding protein 3 (GATA-3) has been proved to work as one of the most important cytokine expression regulators [8]. GATA-3, as a factor of the specific transcription of Th2 cell has its binding sites all cover the promoter regions of Th2 cytokines [9]. Importantly, it has been found that during the menstrual cycle, GATA-3 is expressed cyclically in the human endometrial epithelium [8]. Additionally, Peng et al. [10] suggested that the expression of GATA-3 in endometrial epithelial cells of women with endometriosis was higher than that of healthy women. Specially, it has been proposed that number of proliferating endothelial cells increase in the endometrium of endometriosis patients [11, 12], which implies that the proliferation of endometrial cells is important in the patho- 
genesis of endometriosis. However, to our knowledge, the role of GATA-3 in the proliferation of endometrial cells has not been investigated.

\section{Objectives}

In this study, endometrial tissue was collected from 20 women with endometriosis and 10 women without endometriosis, and the expression of GATA-3 in the endometrium cells of endometriosis patients and normal controls was compared. Further, the proliferation of endometrium cells from endometriosis patients and normal controls was investigated as well. This study may help to provide a deeper understanding on the mechanisms of GATA-3 that is involved in the proliferation of endometrial cells.

\section{MATERIALS AND METHODS}

\section{Sample collection}

A total of 20 patients (aged 37-49) with late stage endometriosis (experimental group) and 10 patients (aged 37-49) with carcinoma in situ of the cervix without endometriosis (control group) were included. At least six months prior to surgery, no patients received any gonadotropin-releasing hormone $(\mathrm{GnRH})$ nor other hormone or anti-inflammatory medication. The endometrial tissues of all patients were obtained through excision or curettage at Shengjing Hospital affiliated toChina Medical University.

The informed consent was signed by all patients or their first-degree relative before operation. Additionally, this study was approved by the Ethics Committee of Shengjing Hospital affiliated to China Medical University and was performed in accordance with the ethical standards. All patients or their first-degree relatives signed an informed consent form prior to operation. Besides, the study was approved by the Ethics Committee of Shengjing Hospital affiliated to China Medical University, and it was carried out based on the ethical standards.

\section{Primary endometrial cell culture and identification}

The endometrial tissue samples from all groups were cut into pieces and digested with $0.1 \%$ type I collagen enzyme for $1 \mathrm{~h}$ at $37^{\circ} \mathrm{C}$. The cell suspension was passed through an 80 mesh sieve, then centrifuged at $850 \mathrm{rpm}$ for $5 \mathrm{~min}$. After removing the supernatant, Dulbecco Modified Eagle Medium (DMEM)/F-12 was added to resuspend cells. Then cell suspension was centrifuged again at $800 \mathrm{rpm}$ for $10 \mathrm{~min}$ to remove neutrophils and erythrocytes. After that, cells were gently resuspended in DMEM/F-12 supplemented with $10 \%$ fetal bovine serum (FBS), and cell suspensions were cultured in flasks at $37^{\circ} \mathrm{C}$ with $5 \% \mathrm{CO}_{2}$. The endometrial cells were identified by immunocytochemical staining of keratin and vimentin.

\section{Groups}

The primary endometrial cells of the experimental and control groups were divided into four groups: the control group into control-no treatment $(\mathrm{C}-\mathrm{N})$, control-negative control (C-NC, transfected with negative control scrambled siRNA), control-lentiviral transfection control (C-LC) and control-GATA-3 lentiviral transfection (C-G3); the experimental group into experiment-no treatment $(\mathrm{E}-\mathrm{N})$, experiment-negative control (E-NC), experiment-lentiviral transfection control (E-LC) and experiment-siGATA-3 lentiviral transfection (E-siG3).

\section{Lentiviral transfection}

The GATA-3 RNA interference (RNAi) lentiviral vector, GATA-3 lentiviral vector, complementary DNA (cDNA) sequent synthesis, and scrambled siRNA negative control were constructed by Shanghai GeneChem Co., Ltd (Shanghai, China). Lentiviral transfection was conducted using a lentiviral vector particle kit purchased from Shanghai GeneChem Co., Ltd (Shanghai, China). The target sequence of siGATA-3 was 5'-AACAUCGACGGUCAAGGCAAC-3' and of the scrambled control was 5'-TTCTCCGAACTGTCACGT-3'. In detail, cells were incubated with the recombinant lentivirus at the optimal multiplicity of infection (MOI) in serum-free DMEM/F-12 containing $5 \mathrm{mg} / \mathrm{mL}$ polybrene at $37^{\circ} \mathrm{C}$ and $5 \% \mathrm{CO}_{2}$. The media was changed after $15 \mathrm{~h}$ of transfection. The transfection efficiency was examined by fluorescence microscopy and flow cytometry after $48 \mathrm{~h}$, and then was confirmed by real-time quantitative polymerase chain reaction (RT-PCR) and western blot.

\section{RT-PCR}

Total mRNA was isolated from cells using Trizol Reagent (Takara, Otsu, Shiga, Japan). First-strand cDNA was synthesized using the PrimeScript First Strand CDNA Synthesis Kit. The SYBR PrimeScript RT-PCR Kit (Takara, Otsu, Shiga, Japan) was used for quantitative PCR with glyceraldehyde-3-phosphatedehydrogenase (GAPDH) as an internal control gene. In this assay the specific primers used were: GATA-3 sense, 5'-CGAGATGGCACGGGACACTA-3', GATA-3 reverse, 5'-TGGTCTGACAGTTCGCACAGG-3'; GAPDH sense, 5'-GCAC CGTCAAGGCTGAGAAC-3', GAPDH reverse, 5'-ATGGTGGTGAAGACGCCAGT-3'. All reactions were performed in the LightCycler 2.0 System $\left(50^{\circ} \mathrm{C}\right.$ for $2 \mathrm{~min}$; $95^{\circ} \mathrm{C}$ for $10 \mathrm{~min} ; 45 \mathrm{cycles}$ of $95^{\circ} \mathrm{C}$ for $15 \mathrm{~s}$ and $60^{\circ} \mathrm{C}$ for $1 \mathrm{~min}$ ). The GATA-3 relative expression value was calculated using the $2^{-\triangle \Delta C T}$ method [12]

\section{Western blot}

Total protein was collected from the cells of the eight groups using a protein extraction kit (Keygen Biotechnology, Nanjing, China). About $50 \mu \mathrm{g}$ of protein was separated by 
$10 \%$ sodium dodecyl sulfate-polyacrylamide gel electrophoresis, and then transferred electrically onto a polyvinylidene fluoride membrane. After being blocked in 5\% non-fat dried milk for $2 \mathrm{~h}$, the membranes were incubated overnight with primary antibodies against either anti-mouse GATA-3 or GAPDH (1:500). Then, the membrane was incubated with horseradish peroxidase-conjugated goat anti-mouse IgG secondary antibody for $4 \mathrm{~h}$. After washing, the signals were developed using an enhanced chemiluminescence kit. To quantify the target protein band intensities (gray-scale values) using Quantity One 1-D Analysis Software.

\section{Cell proliferation assay}

To detect the proliferation of cells, Cell Counting Kit-8 (CCK-8) (Dojindo Laboratories, Kunamoto, Japan) was used following the manufacturer's instructions. Briefly, cell suspension (100 $\mu \mathrm{L} /$ well) was cultured in 96-well culture plates at 5.000 cells/well. After $15 \mathrm{~h}$ of lentiviral transfection, the medium was changed and cells were cultured for another 48,72 or $96 \mathrm{~h}$. Then in each well of the plate, $10 \mu \mathrm{l}$ of the CCK-8 solution was added. After the incubation at $37^{\circ} \mathrm{C}$ with $5 \% \mathrm{CO}_{2}$ for $4 \mathrm{~h}$, the absorbance of each well was measured at $570 \mathrm{~nm}$.

\section{Statistical analysis}

Data were shown as mean \pm standard error (SE). SPSS software version 19.0 was applied to perform all statistical analyses (SPSS Corpoartion, Chicago, IL, USA). Dunnett's t-test and one-way analysis of variance with Student-Newman-Keul analysis were used for statistical comparison. A $P<0.05$ was considered to be statistically significant.

\section{RESULTS}

\section{Relative expression of GATA-3 mRNA}

As shown in Table 1 and Figure $1 \mathrm{~A}$, the relative expression of GATA-3 mRNA in the E-N group was $2.30 \pm 0.03$, which was significantly higher than in the D-N (1.00 \pm 0.10$)$ and E-siG3 group $(0.62 \pm 0.20)(P<0.05)$. In control groups, the relative expression of GATA-3 mRNA in the C-G3 group $(4.64 \pm 0.14)$ was statistically higher than that in the C-N group $(P<0.05)$. No statistically difference was found between the C-NC and C-LC groups or between the E-NC and E-LC groups.

\section{Relative expression of GATA-3 protein}

The relative expression of GATA-3 protein was shown in Table 1 and Figure $1 \mathrm{~B}$ and $1 \mathrm{C}$. When comparing the E-N group $(2.43 \pm 0.12)$ with the $C-N$ group $(1.00 \pm 0.19)$, a significant difference was found between the two groups $(P<0.05)$. When comparing the $\mathrm{C}-\mathrm{N}$ group with the $\mathrm{C}-\mathrm{G} 3$ group, a significantly higher relative expression of GATA-3 protein was found in the $C-G 3$ group $(4.10 \pm 0.51, P<0.05)$. Additionally,
Table 1. The mRNA and protein expressions of GATA-3 measured by real-time fluorescence quantitative polymerase chain reaction and western blot respectively

\begin{tabular}{|l|c|c|}
\hline Groups & $\begin{array}{c}\text { GATA-3 mRNA } \\
\text { expression }\end{array}$ & $\begin{array}{c}\text { GATA-3 protein } \\
\text { expression }\end{array}$ \\
\hline C-N & $1.00 \pm 0.10$ & $1.00 \pm 0.19$ \\
\hline C-NC & $1.17 \pm 0.23$ & $1.13 \pm 0.38$ \\
\hline C-LC & $1.06 \pm 0.18$ & $1.13 \pm 0.36$ \\
\hline C-G3 & $4.64 \pm 0.14$ & $4.10 \pm 0.51$ \\
\hline E-N & $2.30 \pm 0.03$ & $2.43 \pm 0.12$ \\
\hline E-NC & $2.56 \pm 0.58$ & $2.49 \pm 0.33$ \\
\hline E-LC & $2.44 \pm 0.30$ & $2.43 \pm 0.40$ \\
\hline E-siG3 & $0.62 \pm 0.20$ & $1.02 \pm 0.14$ \\
\hline
\end{tabular}

C-G3 - control-GATA-3 lentiviral transfection; C-LC - control-lentiviral transfection control: C-N - control-no treatment; C-NC — control-negative control (transfected with negative control scrambled siRNA); E-LC - experiment-lentiviral transfection control; E-N - experiment-no treatment; E-NC - experiment-negative control; E-siG3 - experiment-siGATA-3 lentiviral transfection; GAPDH - glyceraldehyde3-phosphatedehydrogenase.

there no statistical difference found between the C-NC and $\mathrm{C}-\mathrm{LC}$ groups or between the E-NC and E-LC groups.

\section{Effect of GATA-3 on cell proliferation}

As shown in Figure $2 \mathrm{~A}$ and $2 \mathrm{~B}$, the relative cell proliferation rate of each group obviously increased with time from 48 to 96 h. For intra-group comparison, the relative cell proliferation rates in the C-G3 group at 48,72 and $96 \mathrm{~h}$ were $1.59 \pm 0.05,2.02 \pm 0.01$ and $2.59 \pm 0.05$, respectively, which were significantly higher than that in the C-N, C-NC and C-LC groups $(P<0.05$, Tab. 2 and Fig. $2 A)$. There was no significant difference between the C-N, C-NC and C-LC groups at 48,72 or $96 \mathrm{~h}$. In the experimental group, the relative cell proliferation rate in the E-siG3 group at $48 \mathrm{~h}$ was $1.45 \pm 0.05$, which was significantly lower than that in the E-N (2.02 \pm 0.06$),$ E-NC $(1.97 \pm 0.04)$ and E-LC (2.01 \pm 0.09$)$ groups ( $P<0.05$, Tab. 2 and Fig. 2B). Similar comparative results were found at 72 and $96 \mathrm{~h}$. No significant difference was found between the E-N, E-NC or E-LC groups.

With regard to inter-group comparison, the relative cell proliferation rates in the E-N group at 48,72 and $96 \mathrm{~h}$ were significantly higher than in the $\mathrm{C}-\mathrm{N}$ group $(P<0.05$, Tab. 2$)$.

\section{DISCUSSION}

Endometriosis is an inflammatory disease involving a possible shift towards the Th2-mediated immune response $[13,14]$. As a Th2 cell-specific transcription factor, GATA-3 has been found to play important roles in cell proliferation and differentiation of the mammary glands [15], nephric duct [16] and nervous system [17]. In this study, GATA-3 was up-regulated in the primary endometrial cells of endometriosis patients compared to controls. In addition, the 
A

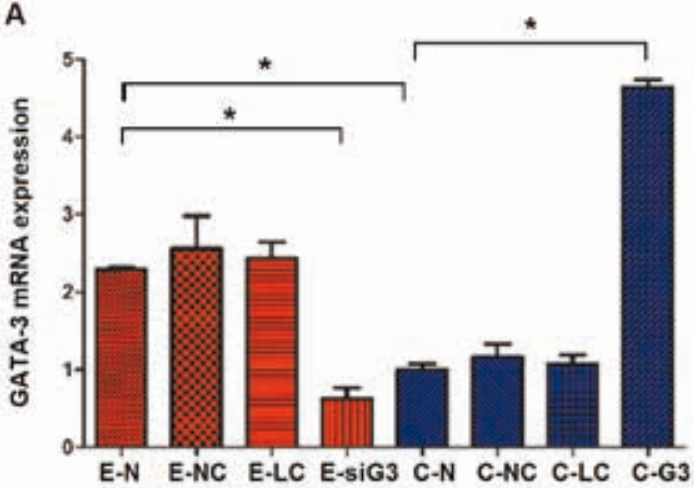

C

\section{GATA-3}

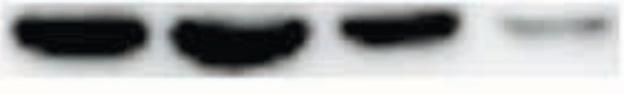

GAPDH

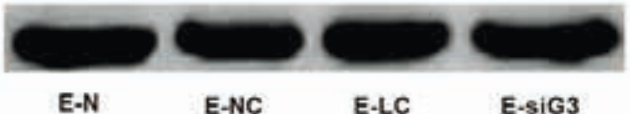

B
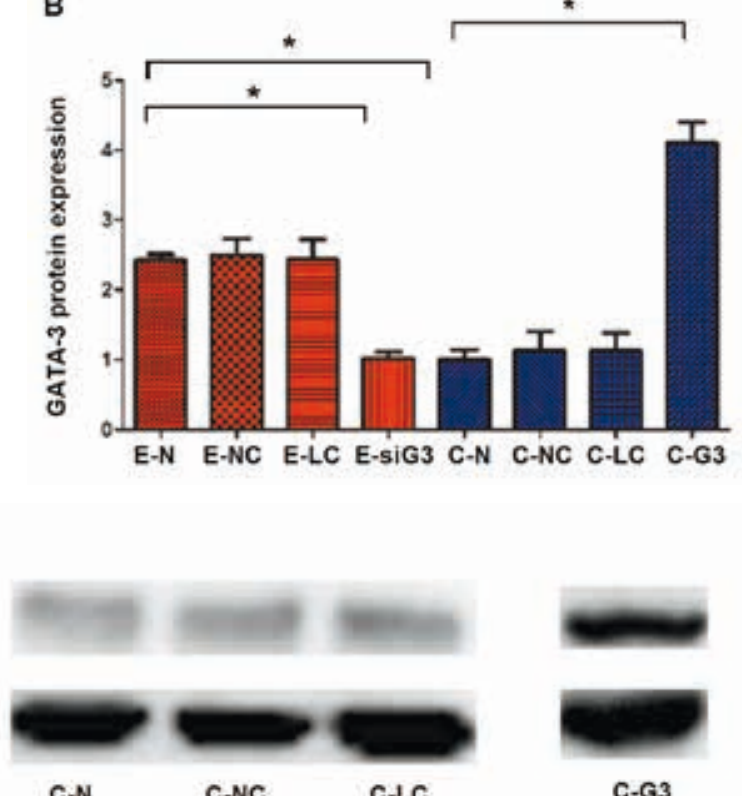

C-G3

Figure 1. The mRNA and protein expressions of GATA-3 were respectively measured by (A) real-time fluorescence quantitative polymerase chain reaction and (B) western blot. (C) The effect of GATA-3 gene silencing and overexpression in primary endometrial cells on GATA-3 protein expression were assayed by western blot. ${ }^{*} P<0.05$

$\mathrm{C}-\mathrm{N}$ - control-no treatment; C-NC — control-negative control (transfected with negative control scrambled siRNA); C-LC - control-lentiviral transfection control; C-G3 - control-GATA-3lentiviral transfection; C-LC - control-lentiviral transfection control; C-N - control-no treatment ; C-NC - controlnegative control (transfected with negative control scrambled siRNA); E-LC — experiment-lentiviral transfection control; E-N — experiment-no treatment; E-NC - experiment-negative control; E-siG3 - experiment-siGATA-3 lentiviral transfection; GAPDH — glyceraldehyde-3-phosphatedehydrogenase

A

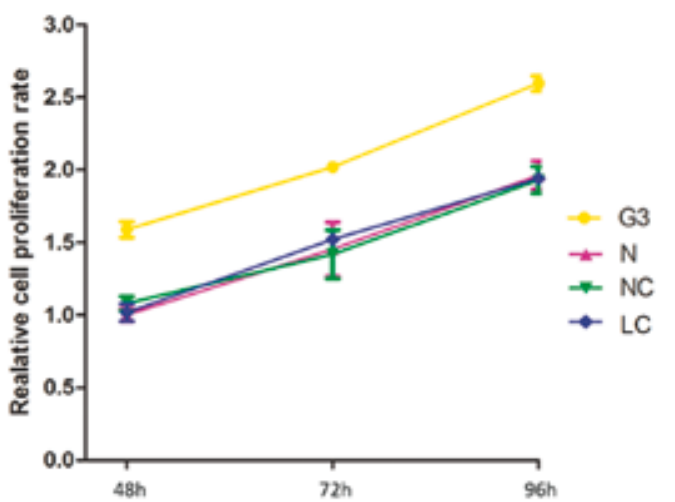

B

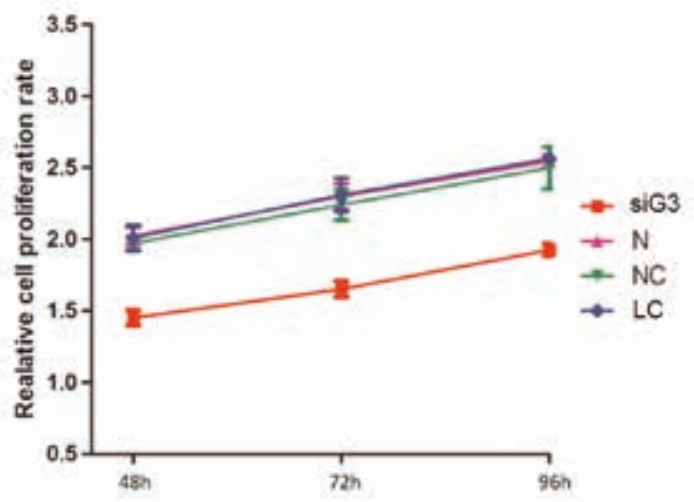

Figure 2. The relative proliferation ability of endometriotic cells in (A) control group and (B) experimental group detected by Cell Counting Kit-8

proliferation rate of endometrial cells in the endometriosis group was significantly higher than in the control group. Importantly, after the endometrial cells of endometriosis patients were transfected with GATA-3 siRNA, the proliferation rate significantly decreased. However, in the control group, when the endometrial cells of were transfected with GATA-3, their proliferation rate increased significantly.

As mentioned above, cytokines play important roles in the immune and menstrual functions of the endometrium [18].
GATA-3 regulates the expression of cytokines in lymphocytes [8]. Some studies have demonstrated the role of GATA-3 in the endometrium throughout the menstrual cycle and in women with endometriosis $[10,19,20]$. Consistent with the findings above, this study revealed increased expressions of GATA-3 mRNA and protein in the primary endometrial cells of endometriosis patients compared to controls. Therefore, GATA-3 may be used as a biomarker in the diagnosis and treatment of patients with endometriosis. 
Table 2. The relative proliferation ability of endometriotic cells at $48 \mathrm{~h}, 72 \mathrm{~h}$, and $96 \mathrm{~h}$ in control group and experimental group detected by Cell Counting Kit-8

\begin{tabular}{|l|c|c|c|}
\hline Groups & $\mathbf{4 8 h}$ & $\mathbf{7 2} \mathbf{h}$ & $\mathbf{9 6} \mathbf{h}$ \\
\hline C-N & $1.00 \pm 0.04$ & $1.45 \pm 0.19$ & $1.96 \pm 0.10$ \\
\hline C-NC & $1.08 \pm 0.05$ & $1.42 \pm 0.16$ & $1.93 \pm 0.09$ \\
\hline C-LC & $1.02 \pm 0.06$ & $1.52 \pm 0.03$ & $2.94 \pm 0.02$ \\
\hline C-G3 & $1.59 \pm 0.05$ & $2.02 \pm 0.01$ & $2.54 \pm 0.04$ \\
\hline E-N & $2.02 \pm 0.06$ & $2.30 \pm 0.09$ & $2.50 \pm 0.15$ \\
\hline E-NC & $1.97 \pm 0.04$ & $2.24 \pm 0.11$ & $2.56 \pm 0.03$ \\
\hline E-LC & $2.01 \pm 0.09$ & $2.31 \pm 0.12$ & $1.92 \pm 0.04$ \\
\hline E-siG3 & $1.45 \pm 0.05$ & $1.65 \pm 0.06$ & \\
\hline
\end{tabular}

C-G3 - control-GATA-3 lentiviral transfection; C-LC - control-lentiviral transfection control; C-N - control-no treatment; C-NC - control-negative control (transfected with negative control scrambled siRNA); E-LC - experiment-lentiviral transfection control; E-N - experiment-no treatment; E-NC - experiment-negative control; E-siG3 - experiment-siGATA-3 lentiviral transfection

It has been suggested that immune mechanisms play an important role in endometriosis development. Studies have widely reported that a variety of abnormalities in immune functions is related to this disease [21]. Specially, not only humoral immunity alterations, but also cell-mediated immunity have been reported in patients with endometriosis [22]. Activated macrophages increase in endometriosis patients and secrete proinflammatory cytokines, such as interleukin-8 (IL-8) and IL-6 [23]. IL-8 promotes the growth and proliferation of endometrial cells [24]. Importantly, David [25] has reported that GATA-3 can promote the differentiation of Th2 cells and induces the expression of Th2 cytokines. Additionally, our previous study (under publication) has suggested that GATA-3 can promote endometrial cells to secrete IL-8; therefore, we speculate that the role of GATA-3 in the proliferation of endometrial cells in patients with endometriosis may be associated with cytokine IL-8.

Furthermore, IL-4, another Th2 cytokine, has been linked to increased mRNA and protein expression in peripheral blood and peritoneal fluid of patients with endometriosis [13, 26, 27]. The action of IL-4 in Th2 development induces the early expression of GATA-3. In turn, the expression of Th2-specific cytokines including IL-4 can also be induced by GATA-3 with the contributions of other transcription factors [28]. Importantly, Zhuo et al. [29] demonstrated that IL-4 stimulated the proliferation of endometriotic stromal cells. Taken together, we speculated that IL-4 may be another important cytokine implicated in the GATA-3-induced proliferation of endometrial cells in endometriosis patients.

\section{CONCLUSIONS}

Our results suggest the role of GATA-3 in promoting proliferation of endometrial cells. Additionally, we also found that blocking GATA-3 can reduce the proliferation of endometrial cells. GATA-3 may be used as a biomarker in the clinical diagnosis and treatment of endometriosis. Further studies are required to elucidate and confirm the exact mechanism.

\section{Acknowledgements}

The present study was supported by The National Natural Science Foundation of China (grant no. 81501235), The Shengjing Hospital of China Medical University (grant no. MF95), and The Natural Science Foundation of Liaoning Province (grant no. 2018010551-301)

\section{Author disclosure and contribiutions}

All authors declare no competing interests.

P.C. acquired funding, designed the study, collected samples, conducted the experiment and statistical analysis and created tables and figures, and wrote the manuscript. F.R. acquired funding and collected samples. DB.W. designed the study and helped in writing the manuscript. All authors have seen and approved the final version of this manuscript.

\section{REFERENCES}

1. Kaponis A, Kollia G, Taniguchi F, et al. Apoptosis in Endometriosis. Endometriosis. 2014: 179-190, doi: 10.1007/978-4-431-54421-0 12.

2. de Ziegler D, Borghese B, Chapron C. Endometriosis and infertility: pathophysiology and management. Lancet. 2010; 376(9742): 730-738, doi: 10.1016/S0140-6736(10)60490-4, indexed in Pubmed: 20801404.

3. Sampson J. Peritoneal endometriosis due to dissemination of endometrial tissue into the peritoneal cavity. American Journal of Obstetrics \& Gynecology. 1927; 14: 93-94.

4. Fauser BC, Diedrich K, Bouchard $\mathrm{P}$, et al. Evian Annual Reproduction (EVAR) Workshop Group 2010. Contemporary genetic technologies and female reproduction. Hum Reprod Update. 2011; 17(6): 829-847, doi: $10.1093 /$ humupd/dmr033, indexed in Pubmed: 21896560.

5. Chen JC, Erikson DW, Piltonen TT, et al. Coculturing human endometrial epithelial cells and stromal fibroblasts alters cell-specific gene expression and cytokine production. Fertil Steril. 2013; 100(4): 1132-1143, doi: 10.1016/j.fertnstert.2013.06.007, indexed in Pubmed: 23849844.

6. Qi X, Qu Y, Nan Z, et al. Caprine endometrial stromal cells modulate the effects of steroid hormones on cytokine secretion by endometrial epithelial cells in vitro. Reprod Biol. 2012; 12(3): 309-315, doi: 10.1016/j. repbio.2012.09.003, indexed in Pubmed: 23153702 
7. Lee SKi, Na BJu, Kim JY, et al. Determination of clinical cellular immune markers in women with recurrent pregnancy loss. Am J Reprod Immunol. 2013; 70(5): 398-411, doi: 10.1111/aji.12137, indexed in Pubmed: 23656517.

8. Inman D, Kawana K, Schust D, et al. Cyclic regulation of T-Bet and GATA-3 in human endometrium. Reprod Sci. 2008; 15(1): 83-90, doi: 10.1177/1933719107309690, indexed in Pubmed: 18212358.

9. Zheng WP, Flavell RA, Zheng W, et al. The transcription factor GATA-3 is necessary and sufficient for Th2 cytokine gene expression in CD4 T cells. Cell. 1997; 89(4): 587-596, indexed in Pubmed: 9160750.

10. Chen P, Zhang Z, Chen Qi, et al. Expression of Th1 and Th2 cytokine-associated transcription factors, T-bet and GATA-3, in the eutopic endometrium of women with endometriosis. Acta Histochem. 2012; 114(8): 779784, doi: 10.1016/i.acthis.2012.01.005, indexed in Pubmed: 22356900.

11. Wingfield $M$, Macpherson $A$, Healy DL, et al. Cell proliferation is increased in the endometrium of women with endometriosis. Fertil Steril. 1995; 64(2): 340-346, indexed in Pubmed: 7542208.

12. Wang DB, Xu YL, Chen P, et al. Silencing Cofilin-1 blocks PDGF-induced proliferation in eutopic endometrium of endometriosis patients. Cell Biol Int. 2013; 37(8): 799-804, doi: 10.1002/cbin.10093, indexed in Pubmed: 23505076 .

13. Podgaec $S, A b r a o$ MS, Dias JA, et al. Endometriosis: an inflammatory disease with a Th2 immune response component. Hum Reprod. 2007; 22(5): 1373-1379, doi: 10.1093/humrep/del516, indexed in Pubmed: 17234676.

14. Chen P, Wang DB, Liang YM. Evaluation of estrogen in endometriosis patients: Regulation of GATA-3 in endometrial cells and effects on Th2 cytokines. J Obstet Gynaecol Res. 2016; 42(6): 669-677, doi: 10.1111/jog. 12957, indexed in Pubmed: 26890586.

15. Kouros-Mehr $\mathrm{H}$, Slorach EM, Sternlicht MD et al. GATA-3 maintains the differentiation of the luminal cell fate in the mammary gland. Cell. 2006; 127(5): 1041-1055, doi: 10.1016/j.cell.2006.09.048, indexed in Pubmed: 17129787.

16. Grote $D$, Souabni A, Busslinger M, et al. Pax 2/8-regulated Gata 3 expression is necessary for morphogenesis and guidance of the nephric duct in the developing kidney. Development. 2006; 133(1): 53-61, doi: 10.1242/dev.02184, indexed in Pubmed: 16319112.

17. Lim KC, Lakshmanan G, Crawford SE, et al. Gata3 loss leads to embryonic lethality due to noradrenaline deficiency of the sympathetic nervous system. Nat Genet. 2000; 25(2): 209-212, doi: 10.1038/76080, indexed in Pubmed: 10835639.
18. Lange M, Misfeldt M. The Importance of TLR3 Expression and Hormonal Regulation of TLR3- Induced Immune Responses in the Human Endometrium. Current Immunology Reviews. 2009; 5(3): 215-226, doi: 10.2 174/157339509788921247.

19. Rubel CA, Franco HL, Jeong JW, et al. GATA2 is expressed at critical times in the mouse uterus during pregnancy. Gene Expr Patterns. 2012; 12(5-6): 196-203, doi: 10.1016/j.gep.2012.03.004, indexed in Pubmed: 22476030.

20. Dyson MT, Roqueiro D, Monsivais D, et al. Genome-wide DNA methylation analysis predicts an epigenetic switch for GATA factor expression in endometriosis. PLoS Genet. 2014; 10(3): e1004158, doi: 10.1371/journal. pgen.1004158, indexed in Pubmed: 24603652.

21. Herington $\mathrm{JL}$, Bruner-Tran $\mathrm{KL}$, Lucas $\mathrm{JA}$, et al. Immune interactions in endometriosis. Expert Rev Clin Immunol. 2011; 7(5): 611-626, doi: $10.1586 /$ eci.11.53, indexed in Pubmed: 21895474.

22. Oral E, Arici A. PATHOGENESIS OF ENDOMETRIOSIS. Obstetrics and Gynecology Clinics of North America. 1997; 24(2): 219-233, doi: 10.1016/s0889-8545(05)70301-6.

23. Giudice LC. Clinical practice. Endometriosis. N Engl J Med. 2010 362(25): 2389-2398, doi: 10.1056/NEJMcp1000274, indexed in Pubmed: 20573927.

24. Paul Dmowski W, Braun DP. Immunology of endometriosis. Best Pract Res Clin Obstet Gynaecol. 2004; 18(2): 245-263, doi: 10.1016/j.bpobgyn.2004.02.001, indexed in Pubmed: 15157641.

25. Farrar JD, Asnagli $\mathrm{H}$, Murphy KM. T helper subset development: roles of instruction, selection, and transcription. J Clin Invest. 2002; 109(4): 431-435, doi: 10.1172/JCl15093, indexed in Pubmed: 11854312.

26. Hsu CC, Yang BC, Wu MH, et al. Enhanced interleukin-4 expression in patients with endometriosis. Fertil Steril. 1997; 67(6): 1059-1064, indexed in Pubmed: 9176444.

27. Antsiferova YS, Sotnikova NYu, Posiseeva LV, et al. Changes in the T-helper cytokine profile and in lymphocyte activation at the systemic and local levels in women with endometriosis. Fertil Steril. 2005; 84(6): 1705-1711, doi: 10.1016/i.fertnstert.2005.05.066, indexed in Pubmed: 16359969.

28. Ouyang W, Löhning M, Gao Z, et al. Stat6-independent GATA-3 autoactivation directs IL-4-independent Th2 development and commitment. Immunity. 2000; 12(1): 27-37, indexed in Pubmed: 10661403.

29. OuYang Z, Hirota Y, Osuga Y, et al. Interleukin- 4 stimulates proliferation of endometriotic stromal cells. Am J Pathol. 2008; 173(2): 463-469, doi: 10.2353/ajpath.2008.071044, indexed in Pubmed: 18599603. 